\title{
Asymptomatic Brain Tumor Detected at Brain Check-up
}

\author{
Masanari OnIZUKA, Kazuhiko SUYAmA, Akira SHIBAYAMA, Tsuyoshi HIURA, \\ Nobutaka HORIE, and Hisaya MIYAZAKI
}

Department of Neurosurgery, Sankoukai Miyazaki Hospital, Isahaya, Nagasaki

\begin{abstract}
Brain check-up was performed in $\mathbf{4 0 0 0}$ healthy subjects who underwent medical and radiological examinations for possible brain diseases in our hospital from April 1996 to March 2000. Magnetic resonance imaging revealed 11 brain tumors which consisted of six meningiomas, three pituitary adenomas, one astrocytoma, and one epidermoid cyst. The detection rate of incidental brain tumor in our hospital was $0.3 \%$. Nine patients underwent surgery, with one case of morbidity due to postoperative transient oculomotor nerve paresis. The widespread use of brain check-up may increasingly detect asymptomatic brain tumors. Surgical indications for such lesions remain unclear, and the strategy for treatment should be determined with consideration of the patient's wishes.
\end{abstract}

Key words: brain check-up, asymptomatic brain tumor, surgical therapy, indication

\section{Introduction}

The brain disease screening system, brain check-up, supports the benefits of early diagnosis for some brain disorders. The detection rate of asymptomatic some brain diseases, such as unruptured aneurysms, has markedly increased with the use of this system in Japan. ${ }^{1,3,7,10-12)}$ However, no clinical studies have investigated the implications for the treatment of incidentally discovered asymptomatic brain tumors. ${ }^{4}$ The present study evaluated the clinical characteristics of asymptomatic brain tumors detected at the brain check-up and discuss the strategy for surgical treatment.

\section{Materials and Methods}

Brain check-up was performed in 4000 people for possible brain diseases at our hospital between April 1996 and March 2000. The 1987 men and 2013 women were aged from 24 to 85 years (mean 56.0 years) and $40.4 \%$ were in the fifth decade. No subject showed any neurological symptoms or signs prior to examination. Magnetic resonance (MR) imaging with a 1.0 Tesla system (Magnetic Impact Expert; Siemens, Erlangen, Bayern, Germany) including axial and coronal fluid-attenuated inversion recovery
(FLAIR) images was routinely performed to detect asymptomatic brain tumors. Patients with arachnoid cyst or angioma were excluded from this study.

\section{Results}

The brain check-up revealed 11 incidental brain tumors in the 4000 subjects. Table 1 shows a summary of the tumors detected which consisted of six meningiomas, three pituitary adenomas, one astrocytoma, and one epidermoid cyst. The patients consisted of six men and five women aged from 49 to 69 years (mean 57.1 years). Nine patients underwent surgery for the tumor, and morbidity occurred due to postoperative transient oculomotor nerve paresis in one patient. The detection rate of incidental brain tumor in our hospital was $0.3 \% .^{5)}$

Meningioma: Six cases of meningioma were identified. Four meningiomas were totally removed, but two patients refused surgical treatment. A patient with a left sphenoid ridge meningioma (Fig. 1) suffered transient left oculomotor nerve paresis postoperatively. One patient with left cerebellopontine angle meningioma underwent repeated MR imaging studies every 6 months, and no tumor growth was observed during the 2-year follow up. The diameter of the accidental meningiomas ranged from

Received August 29, 2000; Accepted July 16, 2001

Author's present address: M. Onizuka, M.D., Department of Neurosurgery, National Nagasaki Medical Center, Omura, Nagasaki, Japan. 
Table 1 Summary of 11 cases of incidentally detected brain tumors

\begin{tabular}{|c|c|c|c|c|c|}
\hline Case No. & Age & Sex & Diagnosis & Treatment & Clinical course \\
\hline 2 & 52 & M & falx meningioma & total removal & good \\
\hline 4 & 49 & $\mathrm{~F}$ & convexity meningioma & total removal & good \\
\hline 5 & 56 & $\mathrm{M}$ & convexity meningioma & follow up & good \\
\hline 6 & 56 & $\mathrm{M}$ & pituitary adenoma & subtotal removal & good \\
\hline 9 & 60 & $\mathrm{M}$ & low grade astrocytoma & total removal & good \\
\hline 10 & 50 & M & epidermoid cyst & total removal & oculomotor nerve paresis \\
\hline 11 & 50 & $\mathrm{~F}$ & cerebellopontine angle meningioma & follow up & good \\
\hline
\end{tabular}

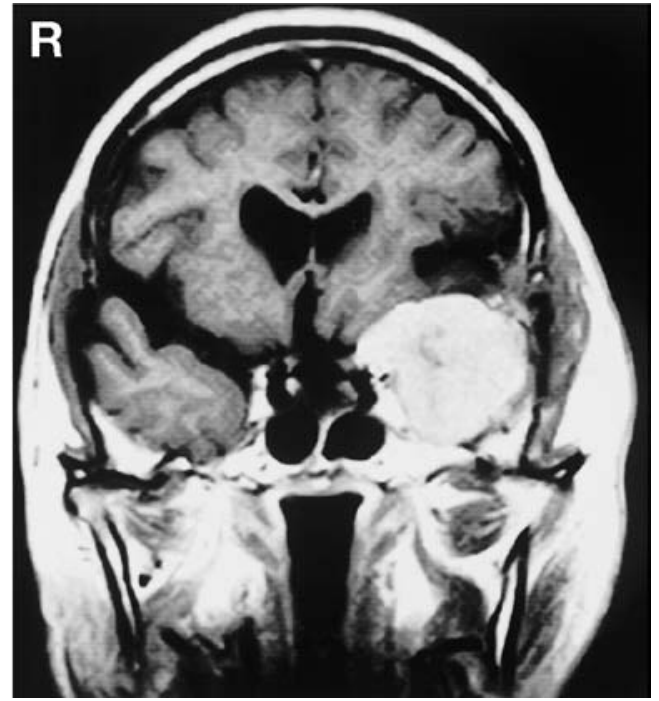

Fig. 1 Case 3. Coronal $T_{1}$-weighted magnetic resonance image with contrast medium showing a large homogeneously enhanced mass (meningioma) in the left temporal fossa.

30 to $45 \mathrm{~mm}$ (mean $34 \mathrm{~mm}$ ).

Pituitary adenoma: Three cases of non-functioning pituitary adenoma with suprasellar extension and compression of the optic chiasma were identified (Fig. 2). MR imaging showed two tumors were solid and the other was cystic. Each patient underwent preoperative endocrine, ophthalmological, and otolaryngological evaluations, which disclosed no abnormal findings. Surgery was performed in all three patients via a transsphenoidal approach.

Glioma: One case of suspected left frontal lowgrade glioma was identified (Fig. 3), but the patient initially refused surgical treatment. A focal seizure occurred after 2 years of follow up, and the patient agreed to surgery. The tumor was completely removed. Histological examination revealed a lowgrade astrocytoma. Postoperatively, the patient was treated with anticonvulsant medication and has had no seizures for a year.

Epidermoid cyst: One case of epidermoid cyst in the prepontine cistern was identified (Fig. 4). The patient developed oculomotor nerve paresis a year later. The tumor was completely removed at another hospital, but the oculomotor nerve paresis did not improve postoperatively.

\section{Discussion}

The present study found 11 cases of asymptomatic brain tumor in 4000 subjects examined over a 4-year period. The detection rate of incidental brain tumors in our hospital was $0.3 \%$, which was in accordance with previous reports.

Meningioma is likely to be the most frequently found tumor at brain check-ups, ${ }^{6,9)}$ and in our study, six meningiomas were found among the 11 tumors detected (54.5\%). Four subjects underwent surgery (mean age 56.0 years). The neurological morbidity of surgery for asymptomatic meningiomas was $23.3 \%$ among patients older than 70 years, but only $3.5 \%$ among younger patients. ${ }^{6)}$ Postoperative morbidity and mortality in asymptomatic patients are not negligible, and poor outcome is associated with more advanced age and with midline tumors.9 Therefore, surgery for asymptomatic meningioma in elderly patients should be considered very carefully, and factors such as location and size of the tumor should be taken into account. ${ }^{8)}$ The tumor size did not increase during a 36.6-month average follow-up period in 43 of 60 cases with asymptomatic meningiomas. ${ }^{6}$ Our strategy for incidental asymptomatic meningiomas is follow up with MR imaging, but most patients chose surgical therapy even after we 

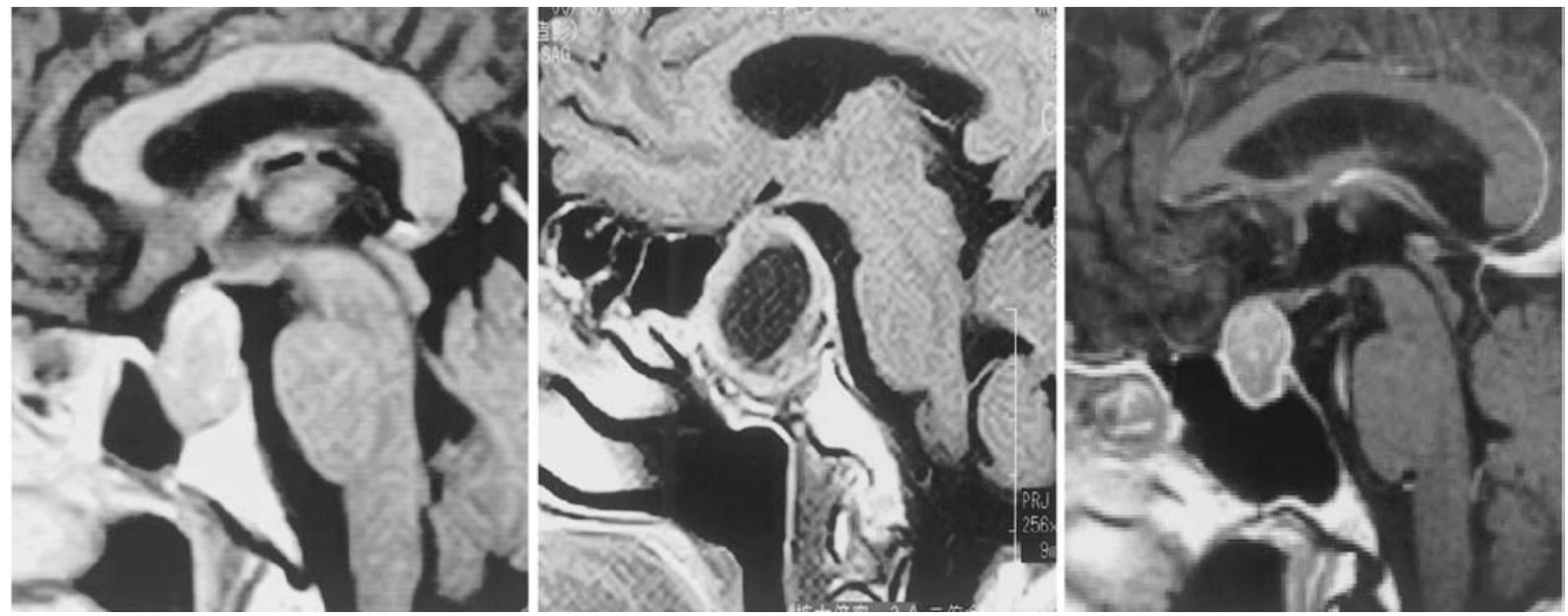

Fig. 2 Cases 6 (left), 7 (center), and 8 (right). Sagittal $T_{1}$-weighted magnetic resonance images with contrast medium showing pituitary adenoma with suprasellar extension and compression of the optic chiasma.
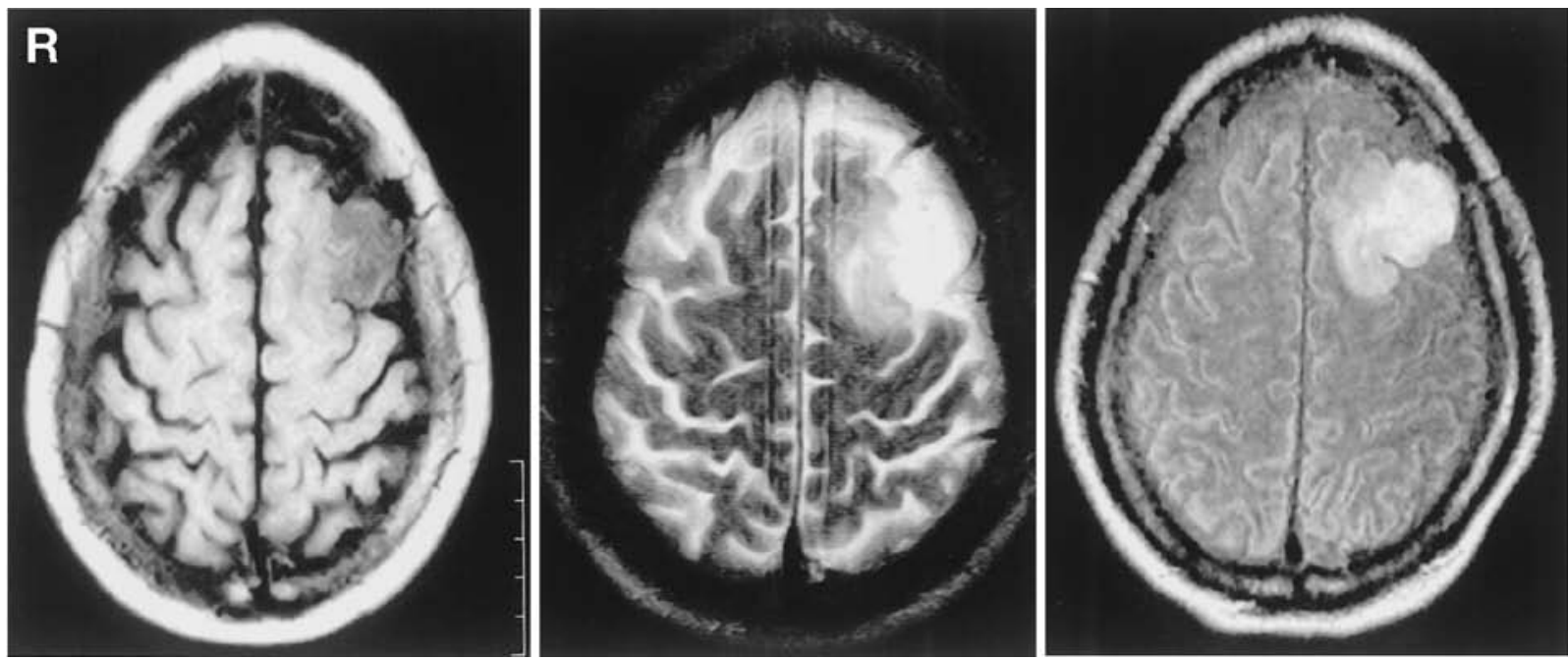

Fig. 3 Case 9. $T_{1}$-weighted (left), $T_{2}$-weighted (center), and fluid-attenuated inversion recovery (right) images showing a mass (low grade astrocytoma) in the subcortical area in the left frontal lobe.

explained our strategy.

The surgical indications for asymptomatic pituitary adenoma are still controversial. ${ }^{10}$ ) When there is no evidence of visual field deficits, medical therapy with a dopamine agonist or follow up with MR imaging are reasonable. At our outpatient's clinic, evidence of tumor enlargement accompanied by compression of the optic chiasma is considered to indicate the need for surgery. In our study, three macroadenomas with compression of the optic chiasma were found, and all three patients agreed to undergo surgery. Solid macroadenomas tend to cause compression symptoms of the optic nerve. ${ }^{2)}$ Solid macroadenoma that has already become symptomatic due to compression of the optic chiasma presents difficulties in surgical manipulation during transsphenoidal surgery, so early surgery might be indicated in patients with a large pituitary adenoma, especially with a solid component. In our study, no microadenomas or parasagittal meningiomas less than $10 \mathrm{~mm}$ in diameter were detected because such small tumors can be missed by axial MR imaging, ${ }^{4,8}$ so coronal images were included to detect such small tumors at the top of the skull or in the sella tur- 


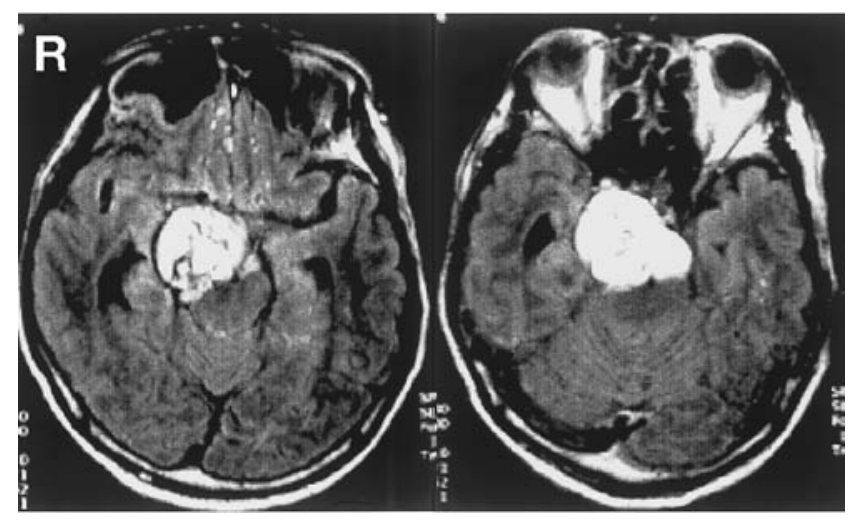

Fig. 4 Case 10. Axial fluid-attenuated inversion recovery images showing an irregularshaped mass (epidermoid cyst) with heterogeneous intensity in the prepontine and chiasmatic cistern and brainstem distortion.

cica from April 2000.

Asymptomatic glioma is rarely found by neuroradiological examination. In our study, only one astrocytoma was found among the 11 tumors detected $(9.1 \%)$. However, three gliomas were found in 1000 volunteers acting as controls for research protocols at the National Institutes of Health, U.S.A. (detection rate of $0.3 \%$ ). ${ }^{5}$ Since the FLAIR image we routinely use at the brain check-up is much more useful for detecting cortical or subcortical lesions near the brain surface than conventional $\mathrm{T}_{2}$-weighted image, ${ }^{3)}$ the incidence of asymptomatic glioma is expected to increase. Surgical treatment is indicated for glioma. ${ }^{4,8)}$

Perioperative morbidity for tumors located in the brain stem or posterior fossa varies markedly among neurological institutions. ${ }^{1)}$ In our series, the epidermoid cyst in the prepontine cistern and chiasmatic cistern was completely removed without worsening the preexisting neurological deficit, and the cerebellopontine angle meningioma was left untreated and followed up by repeated MR imaging. Surgery for small acoustic schwannomas has a better rate of hearing preservation and less facial nerve dysfunction. Surgery is recommended for acoustic schwannomas with moderate compression of the brainstem. The advantages of radiotherapy over conventional surgery for a tumor in cerebellopontine angle in terms of hearing preservation or facial nerve injury are controversial, ${ }^{1)}$ but factors such as the size of the tumor, subject's age, and surgeon's expertise should be considered in deciding the treatment.
The widespread use of the brain check-up may increasingly provide opportunities to detect asymptomatic brain tumors. Surgical indications for such lesions remain unclear, and the strategy for treatment should be determined with consideration of patients' wishes.

\section{References}

1) Anderson T, Loevner L, Bigelow D, Mirza N: Prevalence of unsuspected acoustic neuroma found by magnetic resonance imaging. Otolaryngol Head Neck Surg 122: 643-646, 2000

2) Igarashi T, Saeki N, Yamaura A: Long-term magnetic resonance imaging follow-up of asymptomatic sellar tumors. Neurol Med Chir (Tokyo) 39: 592-599, 1999

3) Ikeda Y, Matsumoto K, Hayashi T, Teramoto A: [Use of fluid-attenuated inversion recovery (FLAIR) images in brain check-up]. No To Shinkei 51: 933-937, 1999 (Jpn, with Eng abstract)

4) Kamiguchi H, Shiobara R, Toya S: Accidentally detected brain tumors: clinical analysis of a series of 110 patients. Clin Neurol Neurosurg 98: 171-175, 1996

5) Katzman GL, Dagher AP, Patronas NJ: Incidental findings on brain magnetic resonance imaging from 1000 asymptomatic volunteers. JAMA 282: 36-39, 1999

6) Kuratsu J, Kochi M, Ushio Y: Incidence and clinical features of asymptomatic meningiomas. J Neurosurg 92: 766-770, 2000

7) Nakagawa T, Hashi K: The incidence and treatment of asymptomatic, unruptured cerebral aneurysms. J Neurosurg 80: 217-223, 1994

8) Nakasu S, Hirano A, Shimura T, Llena JF: Incidental meningiomas in autopsy study. Surg Neurol 27: 319-322, 1987

9) Nishizaki T, Ozaki S, Kwak T, Ito H: Clinical features and surgical outcome in patients with asymptomatic meningiomas. Br J Neurosurg 13: 52-55, 1999

10) Nishizawa S, Ohta S, Yokoyama T, Uemura K: Therapeutic strategy for incidentally found pituitary tumors. Neurosurgery 43: 1344-1350, 1998

11) Obuchowski NA, Modic MT, Magdinec M: Current implications for the efficacy of noninvasive screening for occult intracranial aneurysms in patients with a family history of aneurysms. J Neurosurg 83: 42-49, 1995

12) Yoshimoto T, Mizoi K: Importance of management of unruptured cerebral aneurysms. Surg Neurol 47: 522-525, 1997

Address reprint requests to: M. Onizuka, M.D., Department of Neurosurgery, National Nagasaki Medical Center, 2-1001-1 Kuhara, Omura, Nagasaki 856-8562, Japan.

Commentary on this paper appears on the next page. 


\section{Commentary}

Since multiplication of imaging-diagnosis facilities, patients and neurosurgeons are more and more often confronted with "incidentally-discovered" or "checkup detected" asymptomatic lesions, all over the world. In the authors' series, the detection rate of brain tumors of more than $1 \mathrm{~cm}$ in diameter - with FLAIR images - was $0.3 \%$, that is three a year, for a population of 1000 patients checked per year. Discovering asymptomatic tumors raises a serious medical and ethical problem. Must the patient be pushed to have an operation (or a radiotherapic/radiosurgical treatment) or not? In contrast to patients with asymptomatic aneurysm, who, in our opinion, have to be convinced to undergo radical cure of their malformation to avoid rupture, because of the unpredictability of rupture time and of the potential catastrophic consequences of the rupture, asymptomatic tumors, if they do not grow, may not need any treatment at all. Whatever it might be, the authors have to be congratulated not only for providing the medical community with solid epidemiologic information, but also for the good results that they obtained by surgery in their series. Finally, we agree with the authors' final statement: "Treatment should be determined with consideration of the patients' withes."

Marc P. Sindou, M.D., D.Sc. Professeur of Neurochirurgien Hôpital Neurologique et Neuro-chirurgical Pierre Wertheimer Lyon, France 